OPEN ACCESS

Edited by:

Ralf Jockers,

Université Paris-Sorbonne, France

Reviewed by:

Rami M. El Zein,

INSERM U1016 Institut

Cochin, France

Xiaolong Zhao

Sichuan Provincial People's Hospital,

China

${ }^{*}$ Correspondence:

Lin Sun

xiaosuan2004@126.com

Xia $\mathrm{Li}$

ja_1023@hotmail.com

Shifu Xiao

xiaoshifu@msn.com

†These authors have contributed equally to this work

Specialty section:

This article was submitted to

Endocrinology of Aging,

a section of the journal

Frontiers in Endocrinology

Received: 21 December 2019

Accepted: 06 August 2020

Published: 15 October 2020

Citation:

Li W, Ban C, Yue L, Sun L, LiX and Xiao $S$ (2020) Homozygosity in the APOE 3 Polymorphism Is Associated

With Less Depression and Higher Serum Low-Density Lipoprotein in Chinese Elderly Schizophrenics. Front. Endocrinol. 11:642. doi: 10.3389/fendo.2020.00642

\section{Homozygosity in the APOE 3 Polymorphism Is Associated With Less Depression and Higher Serum Low-Density Lipoprotein in Chinese Elderly Schizophrenics}

\author{
Wei $\mathrm{Li}^{1,2 \dagger}$, Chunxia Ban ${ }^{3 \dagger}$, Ling Yue ${ }^{1,2 \dagger}$, Lin Sun ${ }^{1,2 *}, X_{i a} \mathrm{Li}^{1,2 *}$ and Shifu Xiao ${ }^{1,2 *}$ \\ ${ }^{1}$ Department of Geriatric Psychiatry, Shanghai Mental Health Center, Shanghai Jiao Tong University School of Medicine, \\ Shanghai, China, ${ }^{2}$ Alzheimer's Disease and Related Disorders Center, Shanghai Jiao Tong University, Shanghai, China, \\ ${ }^{3}$ General Psychiatry, Jiading District Mental Health Center, Shanghai, China
}

Background: Depressive symptoms are common comorbidities in schizophrenia. However, the effect of APOE E3 on depressive symptoms has never been investigated in an aging Chinese population with schizophrenia. This cross-sectional study aimed to investigate the effects of APOE E3 on blood lipid metabolism and depressive symptoms in elderly schizophrenics in China.

Methods: Three Hundred and one elderly schizophrenics (161 males, age ranges from 60 to 92 years, with an average age of $67.31 \pm 6.667$ ) were included in the study. Depressive symptoms were assessed using the Geriatric Depression Scale (GDS). APOE gene polymorphism was determined by polymerase chain reaction (PCR). We assessed the correlations of GDS and serum low-density lipoprotein (LDL) with APOE genotypes.

Results: The concentration of LDL in the Homozygous APOE E3 group was significantly higher than that in the non-homozygous APOE E3 group, while the scores of GDS of the Homozygous APOE E3 group were lower than that in the non-homozygous APOE E3 group. Using partial correlation analysis and controlling age, gender, duration of disease, and hyperlipidemia, we found that the scores of GDS were significantly correlated with LDL $(r=-0.194, p=0.016)$.

Conclusions : APOE E3 is associated with less depressive symptoms and higher serum LDL in Chinese elderly patients with schizophrenia, and there is a negative correlation between depressive symptoms and LDL.

Keywords: aging, APOE E3, depressive symptom, Chinese, schizophrenia

\section{INTRODUCTION}

Schizophrenia is associated with an increased prevalence of depressive symptoms. According to previous studies, $\sim 59 \%$ of schizophrenia patients suffer from either minor or major depression $(1,2)$. Depressive symptoms are commonly seen in all stages of schizophrenia, particularly in the acute phase (3). They have been found to correlate with positive symptoms, negative symptoms, 
and general psychopathology in patients with schizophrenia (4). The accumulated evidence from previous studies suggests that the presence of depressive symptoms in patients with schizophrenia has been associated with worse overall outcomes, greater comorbidity, work impairment, poorer quality of life, deterioration of psychosocial functioning, greater risk of relapse, and increased risk of suicide $(5,6)$. It is therefore extremely important to understand the prevalence and influencing factors of depression in schizophrenics and to develop effective interventions.

Various physiological factors increase the risk of depressive symptoms or depression, including lipid metabolism and genetic variations related to lipids (7). A potential genetic variant that affects lipid metabolism symptoms is the Apolipoprotein $\mathrm{E}$ (APOE) gene. APOE is critical in the modulation of phospholipid and cholesterol transport between cells, and it is also believed to play a significant role in neuronal growth and repair (8). Human $\mathrm{APOE}$ is a polymorphic protein, $\mathrm{APOE} \mathrm{E} 2, \mathrm{E} 3$, and $\mathrm{E} 4$, which are encoded by three alleles, $\epsilon 2, \epsilon 3$, and $\epsilon 4$, respectively (9). Metaanalyses of genetic studies reported an association of APOE allele $\epsilon 2$ with major depressive disorder (10). Skoog et al. (11) proved that the presence of APOE allele $\epsilon 4$ predicted future depression and might be an identifier for aging people who are at high risk of clinically significant depression.

The APOE E3 allele is the most frequent allele in all human groups (12) and has been reported to have protection against cardiovascular diseases by maintaining lipid homeostasis (13). However, the effect of APOE E3 on depressive symptoms in schizophrenia has never been investigated. In response, we conducted a cross-sectional study to investigate the effects of homozygosity in the APOE 3 polymorphism on lipid metabolism and depressive symptoms in elderly Chinese schizophrenics.

\section{MATERIALS AND METHODS}

\section{Participants}

This cross-sectional study was conducted between July 1, 2015, and December 31, 2015, and included 301 hospitalized elderly schizophrenics (age ranges from 60 to 92 years, with an average age of $67.31 \pm 6.667$; among them, 161 were males, accounting for $53.5 \%$ ), who were recruited from three mental health centers (including the Shanghai Mental Health Center, the Mental Health Center of Fengxian District in Shanghai, and the Mental Health Center of Jiading District in Shanghai). The method of sampling has been described in our previous studies (14). The inclusion criteria were as follows: (1) aged 60 or more; (2) diagnosed with schizophrenia, which was diagnosed by a senior psychiatrist according to the International Classification of Diseases 10 diagnostic standard; (3) without major medical abnormalities, including unstable, acute, or life-threatening medical illness and central nervous system diseases; (4) was able to cooperate and complete relevant inspections. Subjects with a history of major medical abnormalities (e.g., cancer and infection) and those who chose not to take part were excluded. Through face-toface interviews, we obtained general demographic data (for example, age, education, gender, BMI, duration of disease), daily living habits (smoking, drinking, drinking tea, physical exercise, and hobbies), disease history (hypertension, diabetes, and hyperlipidemia) and currently prescribed medicines (including clozapine, olanzapine, quetiapine, risperidone, and aripiprazole).

This study was approved by the Research Ethical Committee of the affiliated mental health center of the Shanghai Jiaotong University, School of Medicine. Written informed consent was obtained from all participants before the study. All research processes were conducted according to the principles of the Declaration of Helsinki.

\section{Neuropsychological Assessment Depression Evaluation}

Depression is a condition characterized by a depressed mood or loss of pleasure or interest in nearly all activities almost every day for at least 2 weeks (15). The presence of depressive symptoms was determined using the Geriatric Depression Scale (GDS) (16). The GDS consists of 30 items (hereafter referred to as the GDS30 ), and participants were asked to answer "yes" or "no" to these items based on how they felt over the past week, and those with a score higher than 10 points were considered to have depression (17).

\section{Cognitive Assessment}

The Montreal Cognitive Assessment (MoCA) was used to evaluate the cognitive function of these subjects. MoCA is a widely used 10-min cognitive screening test for the detection of mild cognitive impairment (MCI), with high sensitivity (90\%) and specificity (87\%) (18). It has been proven to be effective in detecting cognitive impairment in schizophrenics (19).

\section{Symptom Assessment}

The Positive and Negative Syndrome Scale (PANSS) was utilized to assess the symptoms and severity of schizophrenia, as it is a reliable and valid instrument that has served the scientific research community for decades (20). The PANSS includes four scales measuring positive and negative syndromes, aggressiveness, as well as general severity of illness (21). Several subsequent studies have shown that it has strong psychometric properties in terms of reliability, validity, and sensitivity (22).

\section{Genotyping of APOE and Biochemical Detection of Blood Lipids}

The genomic DNA was removed from peripheral blood (Morning fasting whole blood) by using a Blood Genomic DNA Extraction Kit (spin column, Tiangen Biochemical Science and Technology Co., Ltd., Beijing, China). The APOE genotype was determined by multiplex amplification refractory mutation system polymerase chain reaction (PCR). The multiplex PCR reactions based on the two SNP cores of the APOE gene were measured using the rs7412 and rs429358 design primers as follows: P1:5-'GCCTACAAATCGGAACTGGACAGCT CCTCGGTGCTCTG-3' and P2:5 'TAAGCGGCTCCTCCGCGATGCCCCGGCCTGGTACACTG$3^{\prime}$. We verified the primers on Nucleotide-nucleotide BLAST: (https://ncbiinsights.ncbi.nlm.nih.gov/tag/nucleotideblast). 
According to the methods previously described (23), the 301 subjects were divided into two groups, the Homozygous APOE E3 group $(\varepsilon 3 / \varepsilon 3, n=205)$ and the non-homozygous APOE E3 group $(\varepsilon 2 / \varepsilon 2, \varepsilon 2 / \varepsilon 3, \varepsilon 3 / \varepsilon 4$, and $\varepsilon 4 / \varepsilon 4, n=96)$ group. Tables 1 and 2 list the information about gene distribution in detail. The values of serum triglyceride, cholesterol, fasting blood glucose, lowdensity lipoprotein, and high-density lipoprotein were obtained

TABLE 1 | Allele frequencies and prevalence of APOE among Chinese elderly with schizophrenia.

\begin{tabular}{lccc}
\hline APOE & Male $(\boldsymbol{n}=\mathbf{1 6 1})$ & Female $(\boldsymbol{n}=\mathbf{1 4 0})$ & Combined $(\boldsymbol{n}=\mathbf{3 0 1})$ \\
\hline $\begin{array}{l}\mathrm{E} 2(\epsilon 2 / \epsilon 2, \\
\epsilon 2 / \epsilon 3)\end{array}$ & $23(14.3 \%)$ & $17(12.1 \%)$ & $40(13.3 \%)$ \\
$\mathrm{E} /(\epsilon 3 / \epsilon 3)$ & $109(67.7 \%)$ & $96(68.6 \%)$ & $205(68.1 \%)$ \\
$\mathrm{E} 4(\epsilon 2 / \epsilon 4$, & $29(18.0 \%)$ & $27(19.3 \%)$ & $56(18.6 \%)$ \\
$\epsilon 3 / \epsilon 4, \epsilon 4 / \epsilon 4)$ & & & \\
$\epsilon 2 / \epsilon 2$ & $1(0.6 \%)$ & $1(0.7 \%)$ & $2(0.7 \%)$ \\
$\epsilon 2 / \epsilon 3$ & $22(13.7 \%)$ & $16(11.4 \%)$ & $38(12.6 \%)$ \\
$\epsilon 2 / \epsilon 4$ & $2(1.2 \%)$ & $4(2.9 \%)$ & $6(2.0 \%)$ \\
$\epsilon 3 / \epsilon 3$ & $109(67.7 \%)$ & $96(68.6 \%)$ & $205(68.1 \%)$ \\
$\epsilon 3 / \epsilon 4$ & $23(14.3 \%)$ & $20(14.3 \%)$ & $43(14.3 \%)$ \\
$\epsilon 4 / \epsilon 4$ & $4(2.5 \%)$ & $3(2.1 \%)$ & $7(2.3 \%)$
\end{tabular}

by using the hexokinase method on an auto-analyzer (Dimension Xpand plus).

\section{Statistical Analysis}

Continuous variables were expressed as mean \pm SD and categorical variables were expressed as frequencies (\%). A single sample Kolmogorov-Smirnov test was used to test whether the data conformed to the normal distribution and an independent sample $t$-test was used to compare the data of normal distribution between the Homozygous APOE E3, and the non-homozygous APOE E3 group. The Mann-Whitney $U$ test was used to compare the data of non-normal distribution, while the Chisquare test was used to categorical variables between the two groups. The partial correlation analysis was then used to explore the association between neuropsychological tests and blood lipids (age, education, duration of disease, and hyperlipidemia were controlled). All statistical analyses were performed using SPSS 22.0 (IBM Corporation, Armonk, NY, USA), and two-tailed tests were utilized at a significance level of $P<0.05$.

\section{RESULTS}

Table 1 presents the results of the allele and genotype frequencies. Frequencies of APOE E3 were greatest (68.1\%). Table 2 displays

TABLE 2 | General demographic data of the Chinese elderly with schizophrenia based on APOE E3.

\begin{tabular}{|c|c|c|c|c|}
\hline Variables & Homozygous APOE E3 $(N=205)$ & Non-homozygous APOE E3 $(N=96)$ & $\mathbf{F}$ or $\mathrm{X}^{2}$ & $P$ \\
\hline Age, y & $67.10 \pm 6.542$ & $67.73 \pm 6.888$ & -0.768 & 0.443 \\
\hline Education, y & $8.21 \pm 3.652$ & $7.63 \pm 3.807$ & 1.272 & 0.204 \\
\hline Duration of disease, y & $35.45 \pm 13.241$ & $37.71 \pm 13.066$ & -1.374 & 0.170 \\
\hline $\mathrm{BMI}, \mathrm{kg} / \mathrm{m}^{2}$ & $23.95 \pm 4.217$ & $23.56 \pm 3.989$ & 0.749 & 0.455 \\
\hline Fasting blood glucose, mmol/L & $5.50 \pm 1.491$ & $5.47 \pm 1.264$ & 0.177 & 0.859 \\
\hline Triglyceride, $\mathrm{mmol} / \mathrm{L}$ & $1.38 \pm 0.836$ & $1.39 \pm 0.808$ & 0.076 & 0.927 \\
\hline High density lipoprotein, mmol/L & $1.29 \pm 0.406$ & $1.31 \pm 0.412$ & -0.055 & 0.956 \\
\hline Low density lipoprotein, mmol/L & $2.83 \pm 0.752$ & $2.62 \pm 0.858$ & 2.140 & $0.033^{\star}$ \\
\hline Male, n (\%) & $109(53.2)$ & $52(54.2)$ & 0.026 & 0.902 \\
\hline Hypertension, n (\%) & $78(38.0)$ & $33(34.4)$ & 0.379 & 0.609 \\
\hline Diabetes, n (\%) & $52(25.4)$ & $26(27.1)$ & 0.100 & 0.779 \\
\hline Hyperlipidemia, n (\%) & $72(35.1)$ & $48(50.0)$ & 6.037 & $0.016^{*}$ \\
\hline Smoker, n (\%) & $69(33.7)$ & $28(29.2)$ & 0.604 & 0.509 \\
\hline Drinker, n (\%) & $24(11.7)$ & $9(9.4)$ & 0.364 & 0.693 \\
\hline Tea drinker, n (\%) & $44(21.5)$ & $22(22.9)$ & 0.081 & 0.767 \\
\hline Physical exercise, n (\%) & $66(32.2)$ & $29(30.2)$ & 0.119 & 0.791 \\
\hline Hobby, n (\%) & 78 (38.0) & $31(32.3)$ & 0.938 & 0.369 \\
\hline Clozapine, n (\%) & $33(16.1)$ & 15 (15.6) & 0.011 & 1.000 \\
\hline Olanzapine, n (\%) & $54(26.3)$ & $28(29.2)$ & 0.263 & 0.677 \\
\hline Quetiapine, n (\%) & $26(12.7)$ & $16(16.7)$ & 0.864 & 0.375 \\
\hline Risperidone, n (\%) & $61(29.8)$ & $26(27.1)$ & 0.227 & 0.684 \\
\hline Aripiprazole, n (\%) & 38 (18.5) & $17(17.7)$ & 0.030 & 1.000 \\
\hline MoCA & $14.23 \pm 6.740$ & $12.99 \pm 7.203$ & 1.335 & 0.183 \\
\hline GDS & $9.46 \pm 5.927$ & $11.71 \pm 5.514$ & -2.765 & $0.006^{*}$ \\
\hline PANSS & $63.95 \pm 21.100$ & $65.70 \pm 22.823$ & -0.603 & 0.547 \\
\hline
\end{tabular}

${ }^{*} p<0.05$ 
the characteristic of subjects with different APOE genotypes. By using a single sample Kolmogorov-Smirnov test, we found that BMI $(p>0.05)$ was in the normal distribution, while age, education, duration of disease, fasting blood sugar, triglyceride, high- density lipoprotein, low-density lipoprotein, and scores of MoCA, GDS, and PANSS $(p<0.05)$ were in non-normal distribution.

By using the Independent sample $t$-test (Normal distribution), Mann-Whitney U test (Non-normal distribution), and Chisquare test (Categorical variable), we found that there were statistical differences between the two groups in terms of low density lipoprotein $(p=0.033)$, the score of GDS $(p=0.006)$, and hyperlipidemia $(p=0.016)$. There was no significant difference $(p>0.05)$ in age, education, duration of disease, BMI, fasting blood sugar, triglyceride, high-density lipoprotein, gender, hypertension, diabetes, smoker, drinker, tea drinker, physical exercise, hobby, clozapine, olanzapine, quetiapine, risperidone, aripiprazole, and scores of MoCA and PANSS between the two groups (Table 2). The concentration of LDL in the Homozygous APOE E3 group was significantly higher than the non-homozygous APOE E3 group, while the scores of GDS in the Homozygous APOE E3 group were lower than that in the non-homozygous APOE E3 group. Table 2, Figures 1, 2 show the results. By using partial correlation analysis and controlling age, gender, duration of disease, and hyperlipidemia, we found that the scores of GDS were significantly correlated with low-density lipoprotein $(r=-0.194, p=0.016)$.

\section{DISCUSSION}

To the best of our knowledge, this is the first study to explore the effect of homozygosity in the APOE 3 polymorphism on depressive symptoms in a Chinese elderly population with schizophrenia. The study revealed several interesting findings: (1) that the APOE E3 genotype was associated with less depressive symptoms in elderly patients with schizophrenia; (2) the APOE E3 genotype was associated with higher serum low-density lipoprotein; and, (3) that there was a negative correlation between low-density lipoprotein and depression score.

The present study recruited 301 hospitalized elderly schizophrenics. All participants completed a neuropsychological assessment (MoCA, GDS, and PANSS), APOE gene polymorphism test, and blood lipid test. Using the Mann-Whitney $U$ test, we found that APOE E3 was associated with lower depressive symptoms and higher concentrations of LDL. By using partial correlation analysis and controlling age, gender, duration of disease, and hyperlipidemia, we proved that scores of GDS were significantly correlated with low density lipoprotein $(r=-0.194$, $p=0.016)$.

To verify whether the above conclusions still exist in a non-schizophrenic elderly population, we recruited 154 participants as controls (age ranges from 60 to 78 years,

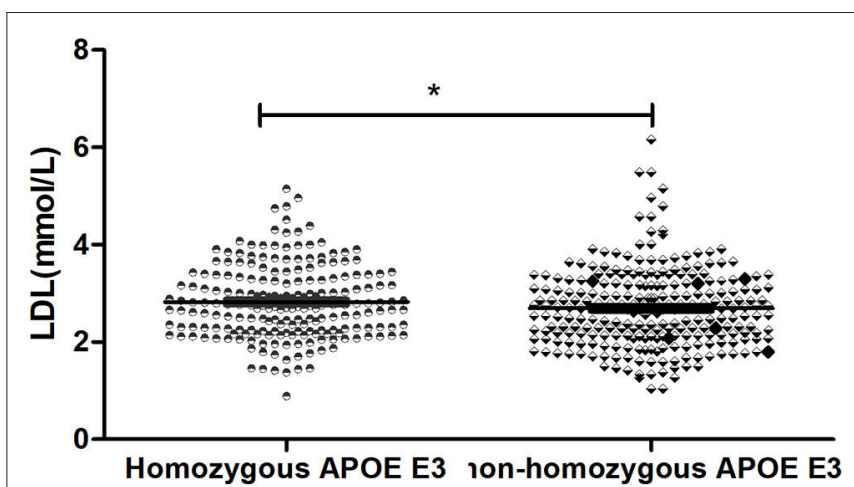

FIGURE 1 | Comparsion of LDL between two groups. *means $p<0.05$.

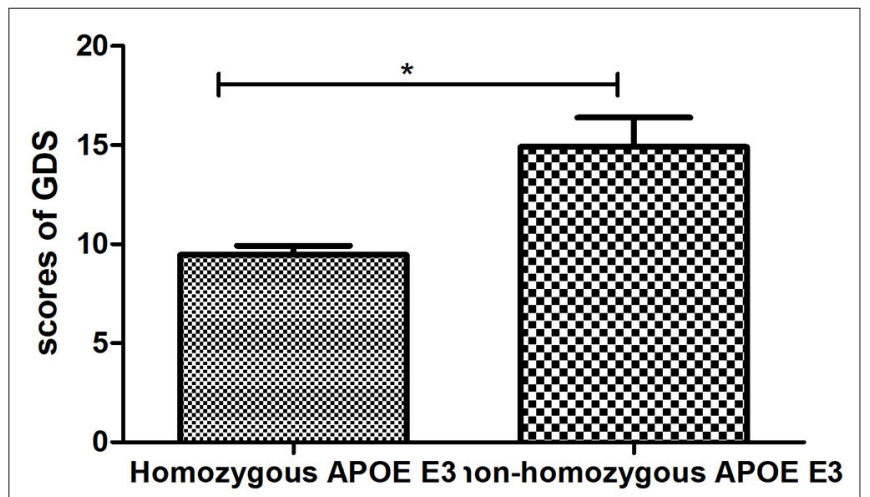

FIGURE 2 | Comparsion of GDS between two groups. * ${ }^{*}$ eans $p<0.05$.

with an average age of $68.54 \pm 6.632$ ). Among them, 72 were male, accounting for $46.8 \%$, and there was no statistical difference in the above indexes between the schizophrenia group and control group. After controlling for age and gender, there was no statistical difference in the concentration of LDL between the Homozygous APOE E3 group and the nonhomozygous APOE E3 group. However, the scores of GDS in the Homozygous APOE E3 group were still lower than those in the non-homozygous APOE E3 group. Table 3 presents these results.

The relationship between APOE gene polymorphism and schizophrenia is very complicated. Gibbons et al. (24) have pointed out that alterations in APOE expression might lead to either the risk of developing schizophrenia or clinical manifestations, as APOE was important in CNS functions such as modulating cell signaling, protein phosphorylation, intraneuronal calcium, and storage-induced synaptic sprouting. However, other related conclusions are not consistent. For example, a French association study and meta-analysis suggested that there was no major role for APOE gene variants in schizophrenia as a whole (25). However, another study supported that the APOE epsilon 4/epsilon 4 genotypes might be associated with early-onset 
TABLE 3 | Characteristics of Chinese normal elderly subjects with different APOE groups.

\begin{tabular}{|c|c|c|c|c|}
\hline Characteristics & Homozygous APOE E3 $(n=97)$ & Non-homozygous APOE E3 $(n=57)$ & $\mathrm{t}$ or $\mathrm{X}^{2}$ & $p$ \\
\hline Age, y & $68.30 \pm 6.515$ & $68.95 \pm 6.867$ & -0.585 & 0.560 \\
\hline Low density lipoprotein, mmol/L & $2.874 \pm 0.870$ & $2.930 \pm 0.809$ & -0.401 & 0.689 \\
\hline Male, n (\%) & $45(46.4)$ & $27(47.4)$ & 0.014 & 1.000 \\
\hline GDS & $4.769 \pm 3.947$ & $6.887 \pm 4.718$ & -2.887 & $0.005^{\star}$ \\
\hline
\end{tabular}

${ }^{*} p<0.05$.

schizophrenia, while the APOE epsilon 3 allele might function protectively in later onset in this disease (26). Therefore, a large sample of longitudinal studies is needed to further examine the association between APOE gene polymorphism and schizophrenia.

It is well-known that lipid metabolism could be affected by the frequency of APOE (27). In our study, we found that APOE E3 was associated with higher LDL in Chinese aging patients with schizophrenia and there was a negative correlation between LDL and depression score. A systematic review and meta-analysis (28) showed that patients with depression tend to have lower LDL (Mean difference $=-4.29,95 \% C I=-8.19$, $-0.40, p=0.03$ ). Beasley et al. (29) found that cholesterol levels were $13 \%$ lower in major depressive disorder $(p=0.018)$ and $10 \%$ lower in bipolar disorder $(p=0.052)$ compared with controls, while there was no significant difference $(p>0.05)$ between schizophrenia and controls. Another study by Olsson et al. (30) also indicated that low LDL might increase the risk of depression and the mechanism might involve immunity, inflammation, and brain dysfunction. However, a previous study suggested that stroke patients with the APOE genotype $\epsilon 3 / \epsilon 3$ had more symptoms of depression compared to the other genotypes (13). Another study showed that there was no significant association between depression and APOE genotype (31). Our findings are partially consistent, and different human populations and different disease types might be responsible for these differences.

Several mechanisms might be used to explain why the APOE E3 genotype helps prevent depression. First, APOE E3 can inhibit amyloid accumulation and increase LDL receptor levels. Second, APOE E3 also plays a crucial role in cholesterol efflux and reverse cholesterol transport, and bears anti-inflammatory and anti-oxidant properties (32). Third, E3 ubiquitin ligase IDOL determines the level of ApoER2 protein in the synapse in response to neuronal activation and regulates the morphogenesis and plasticity of dendritic spines. Furthermore, APOE E3 contributes toward maintaining the integrity of endothelial function and the blood-brain barrier (BBB) at the neurovascular junction (33).

There are some limitations to our research. First, this is a cross-sectional study, unable to establish the causal relationship between APOE E3, LDL, and depressive symptoms. Second, the relatively small sample size reduces the reliability of the study. Third, these subjects have been taking antipsychotics, which could have influenced the research conclusion.

\section{CONCLUSIONS}

The APOE E3 genotype is associated with less depressive symptoms and higher serum low-density lipoprotein in Chinese elderly patients with schizophrenia. There is a negative correlation between depressive symptoms and low-density lipoprotein. Therefore, it may be possible to improve depressive symptoms by increasing blood lipid concentration in patients with schizophrenia, but this conclusion needs to be verified by a large sample of longitudinal research.

\section{DATA AVAILABILITY STATEMENT}

The datasets analyzed in this article are not publicly available, because the database is less than ten years old, and it needs to be authorized by the data owner to be public. Requests to access the datasets should be directed to ja_1023@hotmail.com.

\section{ETHICS STATEMENT}

The studies involving human participants were reviewed and approved by Research Ethical Committee of the affiliated mental health center of Shanghai jiaotong university school of medicine. The patients/participants provided their written informed consent to participate in this study. Written informed consent was obtained from the individual(s) for the publication of any potentially identifiable images or data included in this article.

\section{AUTHOR CONTRIBUTIONS}

WL and LS contributed to the study concept and design. LY and $\mathrm{CB}$ acquired the data. XL and SX analyzed the data and drafted the manuscript. All authors read and approved the final manuscript.

\section{FUNDING}

This work was supported by grants from the National Natural Science Foundation of China (no. 81671402), the National Key R\&D Program of China (2017YFC1310501500), the Cultivation of Multidisciplinary Interdisciplinary Project in Shanghai Jiaotong University (YG2019QNA10), the Clinical research center project of Shanghai Mental Health Center (CRC2017ZD02), Shanghai Clinical Research Center for Mental Health (SCRC-MH) (19MC1911100), and the Feixiang Program of Shanghai Mental Health Center(2020-FX-03). 


\section{REFERENCES}

1. Felmet K, Zisook S, Kasckow JW. Elderly patients with schizophrenia and depression: diagnosis and treatment. Clin. Schizophr. Relat. Psychoses. (2011) 4:239-50. doi: 10.3371/CSRP.4.4.4

2. Xu YM, Li F, Liu XB, Zhong BL. Depressive symptoms in Chinese male inpatients with schizophrenia: prevalence and clinical correlates. Psychiatry Res. (2018) 264:380-84. doi: 10.1016/j.psychres.2018.04.016

3. Baynes D, Mulholland C, Cooper SJ, Montgomery RC, MacFlynn G, Lynch $\mathrm{G}$, et al. Depressive symptoms in stable chronic schizophrenia: prevalence and relationship to psychopathology and treatment. Schizophr. Res. (2000) 45:47-56. doi: 10.1016/S0920-9964(99)00205-4

4. Peitl V, Vidrih B, Karlovic Z, Getaldic B, Peitl M, Karlovic D. Platelet serotonin concentration and depressive symptoms in patients with schizophrenia. Psychiatry Res. (2016) 239:105-10. doi: 10.1016/j.psychres.2016.03.006

5. Majadas S, Olivares J, Galan J, Diez T. Prevalence of depression and its relationship with other clinical characteristics in a sample of patients with stable schizophrenia. Compr. Psychiatry. (2012) 53:14551. doi: 10.1016/j.comppsych.2011.03.009

6. Cotton SM, Lambert M, Schimmelmann BG, Mackinnon A, Gleeson JF, Berk M, et al. Depressive symptoms in first episode schizophrenia spectrum disorder. Schizophr. Res. (2012) 134:20-6. doi: 10.1016/j.schres.2011.08.018

7. Zhou X, Wang J, Lu Y, Chen C, Hu Y, Liu P, et al. Anti-depressive effects of Kai-Xin-San on lipid metabolism in depressed patients and CUMS rats using metabolomic analysis. J Ethnopharmacol. (2020) 252:112615. doi: 10.1016/j.jep.2020.112615

8. Verghese PB, Castellano JM, Holtzman DM. Apolipoprotein E in Alzheimer's disease and other neurological disorders. Lancet Neurol. (2011) 10:24152. doi: 10.1016/S1474-4422(10)70325-2

9. Poirier J, Davignon J, Bouthillier D, Kogan S, Bertrand P, Gauthier S. Apolipoprotein E polymorphism and Alzheimer's disease. Lancet. (1993) 342:697-99. doi: 10.1016/0140-6736(93)91705-Q

10. Lopez-Leon S, Janssens AC, Gonzalez-Zuloeta Ladd AM, Del-Favero J, Claes SJ, Oostra BA, et al. Meta-analyses of genetic studies on major depressive disorder. Mol. Psychiatry. (2008) 13:772-85. doi: 10.1038/sj.mp.4002088

11. Skoog I, Waern M, Duberstein P, Blennow K, Zetterberg H, BörjessonHanson A, et al. A 9-year prospective population-based study on the association between the APOE*E4 allele and late-life depression in Sweden. Biol. Psychiatry. (2015) 78:730-36. doi: 10.1016/j.biopsych.2015.01.006

12. Corbo RM, Scacchi R. Apolipoprotein E (APOE) allele distribution in the world. Is APOE*4 a 'thrifty' allele? Ann. Hum. Genet. (1999) 63(Pt 4):30110. doi: 10.1046/j.1469-1809.1999.6340301.x

13. Leduc V, Domenger D, De Beaumont L, Lalonde D, Belanger-Jasmin S, Poirier J. Function and comorbidities of apolipoprotein e in Alzheimer's disease. Int. J. Alzheimers Dis. (2011) 2011:974361. doi: 10.4061/2011/974361

14. Ban C, Zhang Q, Feng J, Li H, Qiu Q, Tian Y, et al. Low prevalence of lipid metabolism abnormalities in APOE epsilon2-genotype and male patients 60 years or older with schizophrenia. BMC Psychiatry. (2017) 17:399. doi: 10.1186/s12888-017-1530-9

15. Smith K. Mental health: a world of depression. Nature. (2014) 515:181. doi: 10.1038/515180a

16. Lin X, Haralambous B, Pachana NA, Bryant C, LoGiudice D, Goh A, et al. Screening for depression and anxiety among older Chinese immigrants living in Western countries: the use of the Geriatric Depression Scale (GDS) and the Geriatric Anxiety Inventory (GAI). Asia Pac. Psychiatry. (2016) 8:3243. doi: 10.1111/appy.12191

17. Albinski R, Kleszczewska-Albinska A, Bedynska S. Geriatric depression scale (GDS). validity and reliability of different versions of the scale-review. Psychiatr. Pol. (2011) 45:555-62.

18. Nasreddine ZS, Phillips NA, Bedirian V, Charbonneau S, Whitehead V, Collin I, et al. The Montreal Cognitive Assessment, MoCA: a brief screening tool for mild cognitive impairment. J. Am. Geriatr. Soc. (2005) 53:695-99. doi: 10.1111/j.1532-5415.2005.5 3221.x
19. Fisekovic S, Memic A, Pasalic A. Correlation between moca and mmse for the assessment of cognition in schizophrenia. Acta Inform. Med. (2012) 20:186-89. doi: 10.5455/aim.2012.20.186-189

20. Aboraya A, Nasrallah HA. Perspectives on the positive and negative syndrome scale (PANSS): use, misuse, drawbacks, and a new alternative for schizophrenia research. Ann. Clin. Psychiatry. (2016) 28:125-31.

21. Kay SR, Fiszbein A, Opler LA. The positive and negative syndrome scale (PANSS) for schizophrenia. Schizophr. Bull. (1987) 13:261-76. doi: 10.1093/schbul/13.2.261

22. Rabinowitz J, Schooler NR, Anderson A, Ayearst L, Daniel D, Davidson $\mathrm{M}$, et al. Consistency checks to improve measurement with the positive and negative syndrome scale (PANSS). Schizophr Res. (2017) 190:746. doi: 10.1016/j.schres.2017.03.017

23. Donohoe GG, Salomaki A, Lehtimaki T, Pulkki K, Kairisto V. Rapid identification of apolipoprotein $\mathrm{E}$ genotypes by multiplex amplification refractory mutation system PCR and capillary gel electrophoresis. Clin Chem. (1999) 45:143-46. doi: 10.1093/clinchem/45.1.143

24. Gibbons AS, Udawela M, Jeon WJ, Seo MS, Brooks L, Dean B. The neurobiology of APOE in schizophrenia and mood disorders. Front. Biosci. (2011) 16:962-79. doi: 10.2741/3729

25. Schurhoff F, Krebs MO, Szoke A, Loze JY, Goldberger C, Quignon V, et al. Apolipoprotein E in schizophrenia: a French association study and meta-analysis. Am. J. Med. Genet. B Neuropsychiatr. Genet. (2003) 119b:1823. doi: 10.1002/ajmg.b.20007

26. Kampman O, Anttila S, Illi A, Mattila KM, Rontu R, Leinonen $\mathrm{E}$, et al. Apolipoprotein E polymorphism is associated with age of onset in schizophrenia. J. Hum. Genet. (2004) 49:35559. doi: 10.1007/s10038-004-0157-0

27. Guan S, Yang J, Tang Z, Fang X, Wu X, Sun F, et al. The relationship between apolipoprotein (apo) E polymorphism and lipid changes: an 8year cohort study in Beijing elderly persons. Arch. Gerontol. Geriatr. (2012) 55:713-17. doi: 10.1016/j.archger.2011.12.001

28. Persons JE, Fiedorowicz JG. Depression and serum low-density lipoprotein: a systematic review and meta-analysis. J. Affect. Disord. (2016) 206:5567. doi: 10.1016/j.jad.2016.07.033

29. Beasley CL, Honer WG, Bergmann K, Falkai P, Lutjohann D, Bayer TA. Reductions in cholesterol and synaptic markers in association cortex in mood disorders. Bipolar Disord. (2005) 7:449-55. doi: 10.1111/j.1399-5618.2005.00239.x

30. Olsson AG, Angelin B, Assmann G, Binder CJ, Björkhem I, Cedazo-Minguez A, et al. Can LDL cholesterol be too low? Possible risks of extremely low levels. J. Intern. Med. (2017) 281:534-53. doi: 10.1111/joim.12614

31. Lyketsos CG, Baker L, Warren A, Steele C, Brandt J, Steinberg M, et al. Depression, delusions, and hallucinations in Alzheimer's disease: no relationship to apolipoprotein E genotype. J. Neuropsychiatr. Clin. Neurosci. (1997) 9:64-7. doi: 10.1176/jnp.9.1.64

32. Liao F, Yoon H, Kim J. Apolipoprotein E metabolism and functions in brain and its role in Alzheimer's disease. Curr. Opin. Lipidol. (2017) 28:607. doi: 10.1097/MOL.0000000000000383

33. Jofre-Monseny L, Minihane AM, Rimbach G. Impact of apoE genotype on oxidative stress, inflammation and disease risk. Mol. Nutr. Food Res. (2008) 52:131-45. doi: 10.1002/mnfr.200700322

Conflict of Interest: The authors declare that the research was conducted in the absence of any commercial or financial relationships that could be construed as a potential conflict of interest.

Copyright (c) $2020 \mathrm{Li}$, Ban, Yue, Sun, Li and Xiao. This is an open-access article distributed under the terms of the Creative Commons Attribution License (CC BY). The use, distribution or reproduction in other forums is permitted, provided the original author(s) and the copyright owner(s) are credited and that the original publication in this journal is cited, in accordance with accepted academic practice. No use, distribution or reproduction is permitted which does not comply with these terms. 